\title{
A Novel Method for the Evaluation of Fashion Product Design based on Neuro analysis
}

\author{
Baorui Li \\ Shanghai Key Laboratory of \\ Intelligent Manufacturing and \\ Robotics \\ Shanghai University \\ Shanghai, China \\ lbr2013@shu.edu.cn
}

\author{
Kesheng Wang \\ Knowledge Discovery Laboratory \\ Department of Production and \\ Quality Engineering \\ Norwegian University of Science \\ and Technology, \\ Trondheim, Norway \\ kesheng.wang@ntnu.no
}

\author{
Yi Wang \\ School of Materials \\ University of Manchester \\ Manchester, UK \\ yi.wang-2@manchester.ac.uk
}

\begin{abstract}
It is difficult to qualitatively analyze the design effect of the product. This paper will be applying the mathematical fundamentals of the fuzzy theory to create a novel evaluation model. The neuro attributes are used to quantify the factors of affecting the product appearance and the evaluation indicators. This paper will use EEG Emotive and Tobii mobile eye tracking to monitor and acquire subject's brain activity and eye gaze to quantify evaluation factors. This method can scientifically evaluate the uniqueness of product appearance design quality, and provide objective reference for improving product design.
\end{abstract}

Keywords-product design; evaluation method; EEG; eye tracking; fuzzy model

\section{INTRODUCTION}

Evaluating the design effect of the product consists of the process to compare the product design, assess the value of various solutions and select the best design [1]. Effective design evaluation of the product can improve design efficiency and reduce the shortage of the time and resources [2]. The traditional methods that solely on experience and intuition to evaluate the design has been gradually cannot satisfy the development requirements of the product design.

It is necessary to research and develop the more reasonable and advanced theories and methods of evaluation. Neurobased design is an emerging discipline that developed based on the cognitive science and neuroscience [3]. It can use neuroimaging technology combined with the eye tracking technology to study consumer behavior, to explore the mechanism of consumer decision-making on neural activity level and find the real driving force behind the consumer's behavior [4,5]. The concrete method is to use the EEG equipment and eye movement tracking device to monitor and acquire subject's brain activity and eye gaze to quantitative evaluation factors. This method can scientifically evaluate the superiority of product appearance design quality.

The paper is organized as follows. In section 2, the background for evaluation method and neuro-based design is provided. The construction of fuzzy model of evaluation system is discussed in section 3. In section 4, The principle of evaluation method for product design based on neuro-based design is discussed. Case study in section 5 and Conclusions are provided in section 6 .

\section{LITERATURE REVIEW}

\section{A. Evaluation Method for Product Design}

Product design evaluation is an important part of product development. The meaning of the product design evaluation is not only can fully guarantee the quality of the product design, but also reduce the bug in design. The design efficiency was improved by design evaluation [6]. The result of evaluation is an important basis of product quality, production planning and management and investment decisions for manufacturing. The traditional product design evaluation methods are organizing experts by product manufacturers, and then according to the product grade evaluation that submitted by experts to identify outstanding product design.

With the improving of the product design technology, the new product is complicated, the evaluation method has played more important role [7]. Meanwhile, the disadvantages of traditional evaluation methods are subject to design uncertainty, simple indicators and can't from multiple perspectives and more scientific for the product evaluation [8]. Due to the need of product design evaluation, and the continuous development of multidisciplinary cross technology. More and more experts and scholars at home and abroad gradually introduce some new technology and theory to apply in product design evaluation area. Adopting new technologies combined with traditional evaluation methods to evaluate the product design have become the focus in the current [9].

\section{B. Neuro-based design}

The development of neuroscience allowed researchers to dynamically observe human brain activity from different aspects and angles, uncover the direct reasons behind human 
behavior. Neuroscience studies of human brain structure and function of each structure is the physiological basis of neurobased design research [10]. Generally the EEG devices has the characteristics of cheap, low requirement to the experimental environment and easy to popularize, so it become one of the main technical means of neuroscience research [11]. Eye movement instrument as an important equipment of vision research, play an important role in cognitive science and psychology research [12]. Combined EEG signals with eye tracking signals to study the consumer's decision-making process become one of the hot issues at present neuro-based design research.

The EEG signal is the weak electrical potential difference that measured on the scalp when a large number of neurons (Mainly the cone cells) discharge at the same time in the brain neural system [13]. Rami n. Khushaba et. al. [9] choose the Emotiv EPOC to collect the EEG signals when researched people's decision-making process of select preferred crackers according to their own preferences from the crackers described by different characteristics.

Wei-Long Zheng et al. adopts SMI eye tracking glasses to record pupil size changes under different emotional stimuli in the aspects of research human emotional cognitive [14]. In this paper, we choose Tobii eye tracking glasses to record the eyeball fixation time.

\section{THE CONSTRUCTION OF FUZZY MODEL OF EVALUATION SYSTEM}

We usually use the multiple factors to describe a product, and define the main factors of the product as the evaluation factors set $F$, so $F=\left\{f_{1}, f_{2}, \ldots, f_{n}\right\}$. And then the evaluation set be created, This is the evaluation set $\mathrm{S}$ which from the evaluation system to evaluate the each factor of the product, so $S=$ $\left\{s_{1}, s_{2}, \ldots, s_{m}\right\}$. Through the neuro-based design technology to establish the mapping relationship between $\mathrm{F}$ and $\mathrm{S}$.

The specific approach is employ the EEG equipment and eye movement tracking device collecting the subjects' brain activity data and eye gaze time to quantitative evaluation factors. For each $f_{i} \in F, s_{i} \in S$, using the $e_{i j}$ to represent the characteristic indexes of $f_{i}$ on the $s_{i}$ (Possible degree). Obtained a vector $\left(e_{i l}, e_{i 2}, \cdots e_{i m}\right)$ for each $\mathrm{f}_{\mathrm{i}}$ is the feature index vector of $\mathrm{f}_{\mathrm{i}}$ on $\mathrm{S}$, where $i \in(1,2, \cdots n)$. chosen the vector as line to form $m \times n$ matrix, $E=\left(e_{i j}\right)_{m \times n}$. The fuzzy relationship matrix (1) of $\mathrm{F}$ to $\mathrm{S}$ was obtained, called the single factor evaluation matrix.

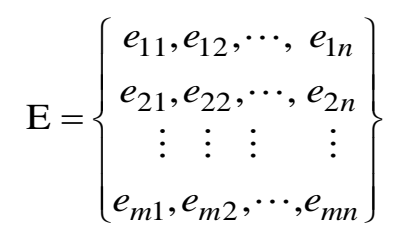

Because of the influence on the effect of each factor to the product focus is different, For the multi-factor evaluation, According to the integral value of the EEG excitement power and the effective observation time by many times experiments and combined with statistical knowledge to distribute the size of the weight of each evaluation factor. the weight can be represented as a fuzzy subset of the $a=\left(a_{1}, a_{2}, \ldots, a_{n}\right)$. The value of 'a' satisfies (2).

$$
\sum_{j=1}^{\mathrm{n}} a_{j}=1
$$

When the fuzzy evaluation matrix ' $\mathrm{E}$ ' and the factorial weight sets 'a' be known, so the comprehensive evaluation model can be defined as $B=a \oplus R$. ' $\oplus^{\prime}$ as the weighted average fuzzy analytic operator; 'B' is the comprehensive evaluation results and the $b_{j}$ represent evaluation objects' membership degree at the $\mathrm{j}$ grade in the evaluation sets. The evaluation corresponding to the largest object from $b_{j}=(j=1,2, \ldots, m)$ as the evaluation conclusion.

\section{THE PRINCIPLE OF EVALUATION METHOD FOR PRODUCT DESIGN BASED ON NEURO-BASED DESIGN}

\section{A. The overall framework}

According to the development of the classic mode S-O-R (Stimulus-Organism-Response) of people's behavior [15, 16], people's behavior can be divided into perception, judgment, decision and action stages. In the face of numerous products, people tend to choose their favorite, so subjects' choice behavior includes a kind of evaluation of the design effect of product. As shown in Fig.1, the principle of evaluation method for product design based on neuro-based design [17]. Using the neuro-based design technology to monitor all choice behavior of the subjects.

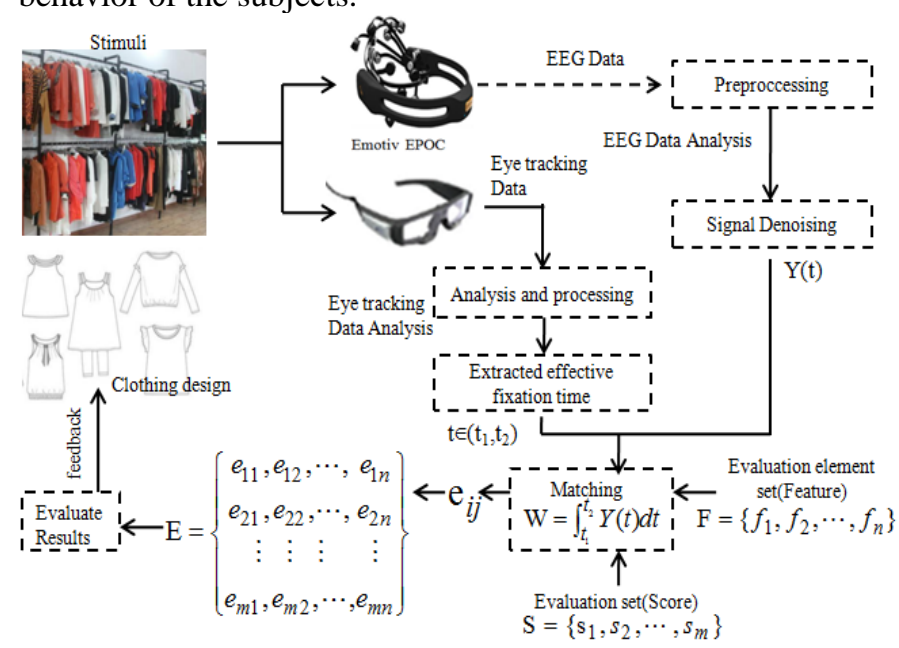

Fig. 1. The principle of fashion design effect evaluation based on neurobased design

\section{B. Data acquisition and processing}

Adopt Emotiv EPOC with 14 channels to collect EEG data, analyzed the EEG data which collected by Emotiv EPOC in the Matlab. Detailed process of EEG signal is shown in Fig.2.Firstly, the collected EEG signals into the signal preprocessing stage. In order to filter the noise and remove the artifacts, the EEG data was processed with a bandpass filter after detrending analysis. In signal denosing stage, adopted the combination of Independent Component Analysis (ICA) 
and discrete wavelet transform (DWT) to clean the EEG signals[18,19].

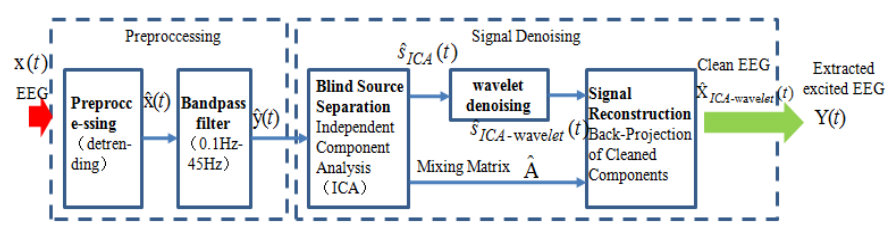

Fig. 2. Detailed process of EEG signal

The clean EEG signal is $\hat{\mathrm{X}}_{I C A \text {-wavelet }}(t)$, then extracted the excited EEG signal for specific factor, Using $Y(t)$ represent the excited EEG signal.

Tobii eye tracking glasses has a very wide range of applications [20]. In this experiment, we selected the Tobii eye tracking glasses to record the subjects' eye movement and fixation points. Participants' eye movement situation will be recorded into the form of a continuous video for storage [21]. According to the subjects' excited situation in the EEG signal when them accept the stimulation of evaluation factor to determine the starting point time $t_{1}$ and the finish point time $t_{2}$. So the subjects' excitement period is $t \in\left(t_{1}, t_{2}\right)$ under the stimulation of the specific factor.

\section{The principle of evaluation}

Chosen $\mathrm{W}=\int_{t_{t}}^{t_{2}} Y(t) d t$ as the basis of evaluation in this study. According to the size of the $W$ value under the situation of repeat experiments combined with expert system to define evaluation levels and the scope of each level $W$ value, Created the evaluation set $\mathrm{S}$. To test each evaluation factor, Measured $Y(t)$ and $t \in\left(t_{1}, t_{2}\right)$ of each evaluation factor. Obtained $W_{i}$ of specified evaluation factor $f_{i}$ by integral formula $\mathrm{w}=\int_{t_{t}}^{t_{2}} Y(t) d t$. Matched the $W_{i}$ with the value range the evaluation level $W$, to determine the level of evaluation factor of $f_{i}$. Statistical analysis many times experiment, to gain the evaluation item $e_{i j}$ of the evaluation elements and constructs the evaluation matrix $\mathrm{E}$.

\section{CASE STUdy}

This paper selects ladies shirts as research object. The object is divided into seven feature elements for study, created the evaluation factors set $F=\left(\right.$ Overall $f_{1}$, Neckline $f_{2}$, Shoulder $f_{3}$, Front skirt $f_{4}$, Cuff $f_{5}$, Waist $f_{6}$, Sweep $f_{7}$ ). The evaluation set was created according to the evaluation system, $S=$ ( Grade A $s_{1}$, Grade $B s_{2}$, Grade $C s_{3}$, Grade $D s_{4}$, Grade $E s_{5}$, Grade $\left.F, s_{6}\right)$. Choose 15 women as subjects whom suit the shirt, the 15 women are from consumer groups of this kind shirt. Adopt Emotiv EPOC and Tobii eye tracking glasses to carry out the experiment. Processing and analysis eye movement data and EEG data to obtain eye movement heat map and brain topographic map, as shown in Fig.3. Eye movement heat map reflects the length of time of the subjects' eye fixed on the characteristic elements in the process of experiment. Brain topographic map reflects the strength of the brain excitability when subjects' eye gaze the characteristics.

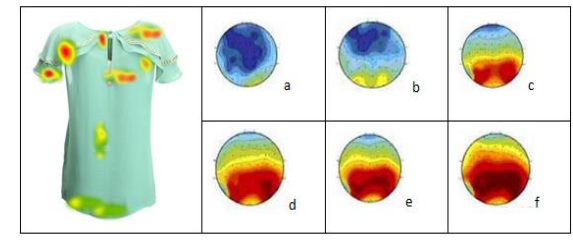

Fig. 3. Eye movement heat map and brain topographic map

Assume that the EEG signals is $\mathrm{Y}(\mathrm{t})$ after processing, and the effective fixation time is $t \in\left(t_{1}, t_{2}\right)$ that extracted by the eye tracking glasses. Then the basis of the feature elements evaluation is $\mathrm{w}=\int_{t_{2}}^{t_{2}} Y(t) d t$. According to repeated experiments combined with the principle of mathematical statistics to divide the scope of each level W.

Tab. 1 is the evaluation test result for the M shirts:

TABLE I. THE TEST RESULT FOR EVERY FEATURE ELEMENTS

\begin{tabular}{|c|c|c|c|c|c|c|c|}
\hline $\mathrm{S}_{\mathrm{F}}$ & $\mathrm{s}_{1}$ & $\mathrm{~s}_{2}$ & $\mathrm{~s}_{3}$ & $\mathrm{~s}_{4}$ & $\mathrm{~s}_{5}$ & $\mathrm{~s}_{6}$ & Weight \\
\hline $\mathrm{f}_{1}$ & $10 / 15$ & $3 / 15$ & $1 / 15$ & $1 / 15$ & 0 & 0 & 0.25 \\
\hline $\mathrm{f}_{2}$ & $9 / 15$ & $4 / 15$ & $2 / 15$ & 0 & 0 & 0 & 0.15 \\
\hline $\mathrm{f}_{3}$ & $8 / 15$ & $5 / 15$ & $1 / 15$ & 0 & $1 / 15$ & 0 & 0.11 \\
\hline $\mathrm{f}_{4}$ & $7 / 15$ & $3 / 15$ & $2 / 15$ & $1 / 15$ & $1 / 15$ & $1 / 15$ & 0.14 \\
\hline $\mathrm{f}_{5}$ & $6 / 15$ & $6 / 15$ & $1 / 15$ & $1 / 15$ & $1 / 15$ & 0 & 0.10 \\
\hline $\mathrm{f}_{6}$ & $9 / 15$ & $3 / 15$ & $2 / 15$ & 0 & 0 & $1 / 15$ & 0.16 \\
\hline $\mathrm{f}_{7}$ & $5 / 15$ & $4 / 15$ & $2 / 15$ & $2 / 15$ & $1 / 15$ & $1 / 15$ & 0.09 \\
\hline
\end{tabular}

The (3) is the evaluation matrix E :

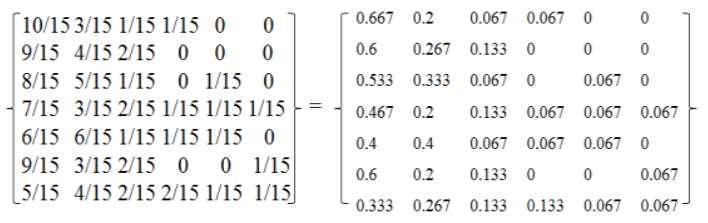

The weights of feature of every feature elements is $a=$ $(0.25,0.15,0.11,0.14,0.10,0.16,0.09)$. Using the weighted average fuzzy analytic operator, It balanced consideration the size of the weights of all factors, and more suitable for the overall optimization. It's suitable for this shirts evaluation, So the mathematical model for comprehensive evaluation is:

$\mathrm{B}=\mathrm{a} \oplus \mathrm{R}=(0.25,0.15,0.11,0.14,0.10,0.16,0.09) \oplus\left\{\begin{array}{llllll}0.667 & 0.2 & 0.067 & 0.067 & 0 & 0 \\ 0.6 & 0.267 & 0.133 & 0 & 0 & 0 \\ 0.533 & 0.333 & 0.067 & 0 & 0.067 & 0 \\ 0.467 & 0.2 & 0.133 & 0.067 & 0.067 & 0.067 \\ 0.4 & 0.4 & 0.067 & 0.067 & 0.067 & 0 \\ 0.6 & 0.2 & 0.133 & 0 & 0 & 0.067 \\ 0.333 & 0.267 & 0.133 & 0.133 & 0.067 & 0.067\end{array}\right\}$

$=(0.547,0.251,0.103,0.045,0.030,0.009)$.

The (4) is the evaluation results: $54.7 \%$ subjects think this shirts is grade $\mathrm{A} ; 25.1 \%$ subjects think this shirts is grade $\mathrm{B}$; $10.3 \%$ subjects think this shirts is grade $C ; 4.5 \%$ subjects think this shirts is grade $\mathrm{D} ; 3.0 \%$ subjects think this shirts is grade $\mathrm{E}$ and only $0.9 \%$ subjects think this shirts is grade F. According to the principle of the maximum membership degree, the final evaluation result of this shirts is Grade A. Meanwhile $A+B+C=90.1 \%$, So we can identify the shirts design is very successful. 


\section{CONCLUSION}

In this paper, the effect of evaluation methods is studied by applying fuzzy logic. The characteristics of the product are analyzed, then a product evaluation factor is established. The evaluation set is created according to the evaluation of product features. Preference degree of subjects for product various characteristics was quantified by neuro-based design means. From the level of the brain response to the product characteristics and the fixation time of eye to product characteristics to analysis subjects' preference degree for the characteristics of each product.

The subjects' evaluation conclusion for product are obtained from the comprehensive evaluation result of each feature in mathematical method. The method will allow the designer to avoid the influence of external factors and subjective factors on the test results. The evaluation accuracy of the product design effect is improved. Provides a more scientific reference for designers to improve their design, meanwhile provide accurate reference for enterprise managers to make production decisions and adjust the product strategy.

\section{REFERENCES}

[1] Varun Tiwaria, Prashant Kumar Jainb\& Puneet Tandon, "Product design concept evaluation using rough sets and VIKOR method", Advanced Engineering Informatics.vol. 30, pp.16-25, January 2016.

[2] Songhua Ma, Zhaoliang Jiang \& Wenping Liu, "Evaluation of a design property network-based change propagation routing approach for mechanical product development", Advanced Engineering Informatics. vol. 30, pp. 633-642, Apirl 2016.

[3] Nick Lee, Amanda J. Broderick \& Laura Chamberlain, "What is "neurobased design'? A discussion and agenda for future research", International Journal of Psychophysiology, vol. 63, pp. 199-204, February 2007.

[4] Hilke Plassmann, Thomas Zoëga Ramsøy \& Milica Milosavljevi, "Branding the brain: A critical review and outlook", Journal of Consumer Psychology, vol. 22, pp. 18-36, January 2012.

[5] Mostafa, M. M. "Brain processing of vocal sounds in advertising: A functional magnetic resonance imaging (fMRI) study", Expert Systems with Applications, vol. 39, pp. 12114-12122, November 2012.

[6] Sawitree Kalakul, Stefano Cignitti, Lei Zhang \& Rafiqul Gani, "Integrated Computer-aided Framework for Sustainable Chemical Product Design and Evaluation", Computer Aided Chemical Engineering, vol. 38, pp. 2343-2348, December 2016.

[7] Yang Xu, Alain Bernard \& Nicolas Perry, "Knowledge evaluation in product lifecycle design and support", Knowledge-Based Systems, vol. 70, pp. 256-267, November 2014.
[8] Khushabaa, R. N., Greenacreb, L., Kodagodaa, S., Louviereb, J., Burkeb, S., \& Dissanayake, G. "Choice modeling and the brain: A study on the Electroencephalogram (EEG) of preferences", Expert Systems with Applications, vol.39, pp. 12378-12388, November 2012.

[9] Rami N. Khushaba, Chelsea Wise, Sarath Kodagoda et al. "Consumer neuroscience: Assessing the brain response to marketing stimuli using electroencephalogram (EEG) and eye tracking", Expert Systems with Applications, vol, 40, pp. 3803-3812, July 2013.

[10] Tony Tien BSc, Philip H. Pucher, MRCS \& Mikael H. Sodergren. PhD. FRCS, Eye tracking for skills assessment and training: systematic review. Journal of Surgical Research, vol. 191, pp. 169-178, September 2014.

[11] Akhtar, M. T., Mitsuhashi, W., \& James, C. J. "Employing spatially constrained ICA and wavelet denoising for automatic removal of artifacts from multichannel EEG data", Signal Processing, vol. 92, pp. 401-416, February 2012.

[12] Michael Plöchl, José P. Ossandón \& Peter König, “Combining EEG and eye tracking: identification, characterization, and correction of eye movement artifacts in electroencephalographic data", Frontiers in Human Neuroscience, October 2012.

[13] E Maris, R Oostenveld, "Nonparametric statistical testing of EEG- and MEG-data", Journal of Neuroscience Methods, vol.164, pp. 177-190 , August 2007.

[14] Wei-Long Zheng, Bo-Nan Dong, Bao-Liang Lu, "Multimodal Emotion Recognition Using EEG and Eye Tracking Data", IEEE Engineering in Medicine and Biology Society, pp:5040-5043, 2014

[15] Babiloni, F., D. Mattia, C. Babiloni, L. Astolfi, S. Salinari, A. Basilisco, et al. "Multimodal integration of EEG, MEG and fMRI data $f$ or the solution of the neuroimage puzzle", Magnetic Resonance Imaging, vol. 22, pp. 71-76, December 2004.

[16] Deppe, M., W. Schwindt, H. Kugel, H. Plassman, and P. Kenning, "Nonlinear responses within the medial prefrontal cortex reveal when specific implicit infor mation influences economic decision making", Journal of Neuroimaging, vol. 15, pp. 171-182, April 2005.

[17] Mostafa, M. M. "Brain processing of vocal sounds in advertising: A functional magnetic resonance imaging (fMRI) study”, Expert Systems with Applications, vol. 39, pp. 12114-12122, November 2012.

[18] M Brunori, A Colosimo, MT Wilson, "EEG signal processing. Computational Intelligence \& Neuroscience”, vol. 2007, pp. 1178-1181, February 2012.

[19] MA Qing-guo \& WANG Xiao-yi, "From Neuroeconomics and Neurobased design to Neuromanagement", Journal of Industrial Engineering Management, vol. 20, pp. 129-132, March 2006.

[20] DU Jian-gang \& WANG Lin, "Research on Neuro-based designintroduction of fMRI", Economic Management Journal in China, vol. 34, pp. 189-199, March 2012.

[21] Hilke Plassmann, Thomas Zoëga Ramsøy \& Milica Milosavljevi, "Branding the brain: A critical review and outlook", Journal of Consumer Psychology, vol. 22, pp. 18-36, January 2012. 